Methods We screened 463 epigenetic regulatory genes using a siRNA library in a high-throughput ISRE-luciferase assay. QPCR and Western blot were used to study the function of targeted genes.

Results Among those genes which significantly enhanced or inhibited ISRE activity, CDK1 had a differential expression in PBMCs and renal biopsies between SLE patients and healthy controls and was positively correlated with "IFN scores" and disease activities of SLE patients. We revealed that the expression of ISGs and phosphorylation of STAT1 could be reduced by knocking down CDK1, while over-expression of CDK1 produced an opposite effect. As specific inhibitors of IFN pathway has emerged as a promising treatment for SLE and CDK1 inhibitors are being on trial for some types of cancer, we wondered whether CDK1 inhibitor could be a potential repositioning drug for SLE. We found a CDK1 inhibitor, RO3306, could alleviate FN in PBMCs from 5 SLE patients who had high IFN scores. We found that RO-3306 significantly reduced ISGs expression in these cells. Our preliminary data further showed that RO-3306 could reduce proteinuria in SLE mouse model.

Conclusions We proposed that CDK1 is a novel positive regulator of IFN signalling pathway, over-expression of CDK1 in SLE might contribute to the over-activated IFN signalling and inhibition of CDK1 could be used to interfere abnormal IFN signalling in SLE.

\section{A MOLECULAR SIGNATURE BASED ON IFN GENE SIGNATURE AND SEROLOGY DEFINES TWO POPULATIONS OF PATIENTS WITH DIFFERENT BASELINE DISEASE ACTIVITY}

${ }^{1} \mathrm{M}$ Petri ${ }^{\star},{ }^{2} \mathrm{~K}$ Kalunian, ${ }^{3} \mathrm{M}$ Urowitz, ${ }^{4} \mathrm{R}$ Furie, MA Morgan-Cox ${ }^{5},{ }^{\mathrm{M}}$ Silk, ${ }^{7} \mathrm{E}$ Dow, ${ }^{5} \mathrm{R}$ Higgs, ${ }^{5} \mathrm{~S}$ Watts, ${ }^{8} \mathrm{D}$ Isenberg, ${ }^{9} \mathrm{M}$ Linnik. 'Johns Hopkins University School of Medicine, Rheumatology, Baltimore, USA; ${ }^{2}$ University of California- San Diego School of Medicine, Rheumatology, San Diego, USA; ${ }^{3}$ University of Toronto Faculty of Medicine, Rheumatology, Toronto, Canada; ${ }^{4}$ Hofstra North Shore-LIJ School of Medicine, Rheumatology, Great Neck, USA; ${ }^{5}$ Eli Lilly, Statistics, Indianapolis, USA; ${ }^{6}$ Eli Lilly, Immunology, Indianapolis, USA; ${ }^{7}$ Eli Lilly, Bioinformatics, Indianapolis, USA; ${ }^{8}$ University College London, Rheumatology, London, UK; ${ }^{9}$ Lilly Biotechnology Centre, Immunology, San Diego, USA

\subsection{6/lupus-2017-000215.24}

Background and Aims Randomised controlled trials in SLE have shown that response to treatment is influenced by baseline disease activity. The current investigation used objective molecular and biochemical baseline parameters to characterise SLE patients in two large multinational trials $(n=2262$ patients).

Methods Patients were categorised with four dichotomous baseline parameters. SLE(+) was defined by any of the following: IFN signature (high), anti-dsDNA (+), C3 (low) and/or C4 (low). SLE(-) required all of the following: IFN signature (normal), anti-dsDNA (-), C3 (normal) and C4 (normal).

Results Baseline RNA transcript data were available for 1747 of 2262 patients. When IFN status was combined with the serology criteria, 1500 (86\%) were classified as SLE(+) and $247(14 \%)$ were classified as SLE(-). At baseline, SLE(-) patients had significantly lower mean SLEDAI scores (8.3) compared to $\operatorname{SLE}(+)$ (10.7). Baseline SLEDAI $<10$ was observed in $72 \%$ of SLE(-) compared to $38 \%$ of SLE(+). The proportion on corticosteroids at baseline was $49 \%$ in SLE(-) compared to $78 \%$ in $\operatorname{SLE}(+)$; the proportion on immunosuppressants at baseline was $31 \%$ in SLE(-) compared to $44 \%$ in
SLE(+). In the US, $22 \%$ were SLE(-) compared to $10 \%$ for Latin America, $7 \%$ for Europe, and 5\% for ROW.

Conclusions A subset of clinical trial patients was identified using biochemical and molecular markers with high sensitivity for SLE. Seronegative SLE patients with normal IFN gene signature had lower disease activity and were taking less background medication at baseline, two factors which have been negatively associated with response to treatment in some previous trials.

\section{STIMULATOR OF INTERFERON GENES (STING) PLAYS A CRUCIAL ROLE IN TYPE-I IFN PRODUCTION INDUCED BY THE SERA FROM SLE PATIENTS}

Y Kato*, A Kumanogoh, H Takamatsu, J Park. Osaka University, Dept. of Respiratory Medicine- Allergy and Rheumatic Disesase- Graduate School of medicne, Suita- Osaka, Japan

\subsection{6/lupus-2017-000215.25}

Background and Aims Type-I interferon (IFN-I) plays important roles in the pathogenesis of SLE. It has been reported that serum IFN-I levels are high in active SLE patients and that IFN-I is produced when DNA sensors recognise DNAcontaining immune complex. Stimulator of interferon genes (STING) is known as a key molecule in cytosolic DNA-sensing, which leads to IFN-I production. However, the involvement of STING in the pathogenesis of SLE has not been clarified. We studied the role of STING in the production of IFN-I in SLE.

Methods We evaluated both the IFN-I bioactivity in sera and the serum-mediated type-I IFN-inducing activity (IFN-I-IA) in SLE by using two different reporter cell lines. Also, to address contribution of STING in the production of IFN-I, we established the STING-deficient reporter cell lines (STING-KO) using the CRISPR/Cas9 system.

Results IFN-I bioactivity was high in the sera from SLE compared with other autoimmune diseases and healthy controls. Serum-induced IFN-I-IA was also higher in SLE than those in other autoimmune diseases. These reporter cell lines do not respond to the ligands of Toll like receptor (TLR) 8 or TLR9, suggesting the existence of TLR8/9-independent IFN-I-inducing mechanism. Consistently, the enhanced IFN-I-IA in SLE was reduced in the STING-KO, indicating that STING is involved in the serum-induced IFN-I production.

Conclusions Our finding suggests that IFN-I bioactivity is high in the sera of SLE, and that these sera have a potential to induce IFN-I production through STING.

\section{Parallel Session 9: The mosaic of autoimmunity}

\section{CLINICAL SIGNIFICANCE OF AUTOANTIBODIES IN MYOSITIS WITH INTERSTITIAL LUNG DISEASE}

${ }^{1} \mathrm{~T}$ Mimori ${ }^{*}$. 'Kyoto Univerisity, Department of Rheumatology and Clinical Immunology, Kyoto, Japan

10.1136/lupus-2017-000215.26

Interstitial lung disease (ILD) is the most frequent organ involvement found in near half of myositis patients, but it 
reveals various clinical course and therapeutic responsiveness according to the clinical and serological subsets. Some myositis-specific autoantibodies (MSAs) are useful markers for the classification of ILD in myositis and give useful information for predicting the prognosis and determining treatment.

Anti-aminoacyl-tRNA synthetases are closely associated with a common clinical manifestation, termed "anti-synthetase syndrome" including high prevalence of ILD. ILD in patients with anti-synthetases shows a similar clinical course with a favourable response to therapy but frequent recurrences. Therefore, the concomitant use of glucocorticoids and immunosuppressive drugs is recommended from early stage of the disease.

Anti-MDA5 antibody was reported to be associated with clinically amyopathic dermatomyositis (CADM) with rapidly progressive ILD, especially in eastern Asian population. Because of a very poor life prognosis, the intensive immunosuppressive therapy against ILD from early stage is recommended using the combination of high dose glucocorticoids, calcineurin inhibitors and intravenous cyclophosphamide. We have experienced the effectiveness of plasmapheresis in some anti-MDA-positive patients with intractable ILD, suggesting a possible pathogenicity of anti-MDA5 antibody.

Thus, ILD in myositis is dependent on the autoantibodies, therefore it is important to know the profiles of MSAs in PMDM patients. Recently, we established the ELISA systems for anti-synthetase and anti-MDA5 antibodies, which are as efficient as the standard immunoprecipitation assays. These systems enable easier and wider use in the diagnosis and therapeutic decision of patients suspected to have myositis and ILD.

\section{THE INTERNATIONAL CONSENSUS ON STANDARDIZED NOMENCLATURE OF ANTINUCLEAR ANTIBODY HEP-2 CELL PATTERNS (ICAP) INITIATIVE - ITS IMPACT AND UPDATE FROM 3RD ICAP}

${ }^{1} \mathrm{E}$ Chan*, 2J Damoiseaux, OG Carballo ${ }^{3},{ }^{4} \mathrm{~K}$ Conrad, ${ }^{5} \mathrm{~W}$ de Melo Cruvinel, PLCFrancescantonio ${ }^{6}{ }^{7} \mathrm{M}$ Fritzler, ${ }^{8}$ I Garcia-De La Torre, ${ }^{9} \mathrm{M}$ Herold, ${ }^{10} \mathrm{~T}$ Mimori, ${ }^{11} \mathrm{M}$ Satoh, ${ }^{12} \mathrm{C}$ von Mühlen, ${ }^{13} \mathrm{~A}$ Luis. ${ }^{1}$ USA; ${ }^{2}$ Maastricht University Medical Centre, Central Diagnostic Laboratory, Maastricht, The Netherlands; ${ }^{3}$ Hospital Carlos G. Durand, Laboratory of Immunology, Buenos Aires, Argentina; ${ }^{4}$ Technical University of Dresden, Institute of Immunology, Dresden, Germany; ${ }^{5}$ Pontificia Universidade Católica de Goiás, Biology, Goiânia, Brazil; ${ }^{6}$ Pontifícia Universidade Católica de Goiás, Medicine, Goiânia, Brazil; 'University of Calgary, Medicine, Calgary, Canada; ${ }^{8}$ University of Guadalajara, Immunology and Rheumatology, Guadalajara, Mexico; ${ }^{9}$ Medical University of Innsbruck, Internal Medicine VI, Innsbruck, Austria; ${ }^{10}$ Kyoto University, Rheumatology and Clinical Immunology, Kyoto, Japan; ${ }^{11}$ University of Occupational and Environmental Health, Clinical Nursing, Kitakyushu, Japan; ${ }^{12}$ Brazilian Society of Autoimmunity, Immunology, Porto Alegre, Brazil; ${ }^{13}$ Universidade Federal de São Paulo, Rheumatology, Sao Paulo, Brazil

10.1136/lupus-2017-000215.27

Background and Aims The indirect immunofluorescence (IIF) pattern in ANA test provides initial assessment of autoantibody responses in candidate patients at risk of developing SLE. The International Consensus on ANA Patterns (ICAP) Initiative workshop originated in 2014, followed by the $2^{\text {nd }}$ and $3^{\text {rd }}$ workshops in 2015 and 2016. The goal of ICAP is to provide guidelines for ANA interpretation, thereby optimising adoption in diagnostic laboratories and extended to patient care.

Methods A working committee addressed collective issues on ANA nomenclature that are raised by participants representing research, clinical, and diagnostic laboratories.

Results ANA patterns are separated into three major categories (nuclear, cytoplasmic, and mitotic patterns). A total of 28 patterns were defined, described in detail, designated with alpha-numeric codes (AC-1 to AC-28), and summarised under a nomenclature and classification tree (ANApatterns.org). ICAP initiatives include translation of the website into other languages, establishing guidelines in ANA reporting, and programs for continuing education. The translation initiative promotes the establishment and dynamic engagement of a worldwide network. To date, the website displays its content in English, Spanish, Portuguese, Italian and German, while Chinese, Japanese, French, and Greek translations are ongoing. ICAP has provided a common platform to address issues that are of great interest to the scientific community. The establishment of a consensus on ANA reporting will require interaction with committees in charge of establishing disease classification and diagnostic criteria.

Conclusions Future goals include building collaborative data on ANA patterns, establishing new consensus patterns, and developing an interpretative description for each pattern for their widespread clinical use.

\section{DRIVERS OF THE SLE RESPONDER INDEX (SRI-5) ENDPOINT IN CLINICAL TRIALS OF SLE}

${ }^{1} \mathrm{~K}$ Kalunian, ${ }^{*}{ }^{2} \mathrm{M}$ Urowitz, ${ }^{3} \mathrm{D}$ Isenberg, ${ }^{4} \mathrm{~J}$ Merrill, ${ }^{5} \mathrm{M}$ Petri, ${ }^{6} \mathrm{R}$ Furie, MA Morgan$\mathrm{Cox}^{7},{ }^{7} \mathrm{R}$ Taha, ${ }^{8} \mathrm{M}$ Silk, ${ }^{9} \mathrm{M}$ Linnik. ${ }^{1}$ University of California- San Diego School of Medicine, Medicine, San Diego, USA; ${ }^{2}$ University of Toronto Faculty of Medicine, Medicine, Tornoto, Canada; ${ }^{3}$ University College London, Rheumatology, London, UK; ${ }^{4}$ University of Oklahoma College of Medicine, Rheumatology, Oklahoma City, USA; ${ }^{5}$ Johns Hopkins University School of Medicine, Rheumatology, Baltimore, USA; ${ }^{6}$ Hofstra North Shore-LIJ School of Medicine, Rheumatology, Great Neck, USA; ' Eli Lilly, Statistics, Indianapolis, USA; ${ }^{8}$ Eli Lilly, Immunology, Indianapolis, USA; ' ${ }^{2}$ illy Biotechnology Centre, Immunology, San Diego, USA

\subsection{6/lupus-2017-000215.28}

Background and Aims SRI is a composite endpoint used in many SLE trials. The current investigation sought to identify those SRI components that drive response.

Methods This study evaluated data from two large multinational trials that used SRI-5 as primary endpoint $(n=2262$ patients).

Results The overall SRI-5 response rate was 32.8\%. Nonresponse due to lack of a 5-point SLEDAI improvement, conmed violation or dropout were observed in 31\%, 16.5\% and $19.1 \%$, respectively. In contrast, only $0.5 \%(11 / 2262)$ of patients failed to respond due to deterioration by BILAG or PGA, once achieving the first three components.

The most common SLEDAI organ systems involved at baseline were mucocutaneous (90.6\%), musculoskeletal (82.9\%) and immunologic $(71.6 \%)$. The 6 other SLEDAI-defined organ systems were active in $\leq 11 \%$ of patients.

At least one SLEDAI manifestation with low prevalence and high score ( $\geq 4$ pts) was present in $18.1 \%$ and $17.2 \%$ of patients in Trials 1 and 2, respectively. SRI-5 response rates for these patients were higher, regardless of treatment assignment.

Conclusions The SRI-5 response is driven by SLEDAI improvement, concomitant medication adherence and trial completion. Patients with less frequent, more severe manifestations had high placebo response rates. A simple dichotomous improvement in SLEDAI score, coupled with successful trial completion and medication stability, provides a more efficient, clinically relevant approach to assess outcome. 